OPEN ACCESS

Edited by:

Gong-Ping Liu,

Huazhong University of Science and Technology, China

Reviewed by:

Jiaming Liu,

Wenzhou Medical University, China

Yi Hao,

Huazhong University of Science

and Technology, China

*Correspondence:

Shaochang Wu

shaochang_wu@163.com

${ }^{\dagger}$ These authors have contributed equally to this work

Specialty section:

This article was submitted to

Molecular Medicine,

a section of the journal

Frontiers in Cell and Developmental

Biology

Received: 20 November 2020

Accepted: 31 December 2020

Published: 28 January 2021

Citation:

Ling Z, Zhu M, Liu X, Shao L, Cheng $Y$, Yan $X$, Jiang $R$ and Wu S

(2021) Fecal Fungal Dysbiosis in Chinese Patients With Alzheimer's

Disease.

Front. Cell Dev. Biol. 8:631460. doi: 10.3389/fcell.2020.631460

\section{Fecal Fungal Dysbiosis in Chinese Patients With Alzheimer's Disease}

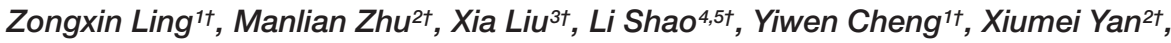 \\ Ruilai Jiang ${ }^{2}$ and Shaochang $W u^{2 *}$
}

\begin{abstract}
'State Key Laboratory for Diagnosis and Treatment of Infectious Diseases, Collaborative Innovation Center for Diagnosis and Treatment of Infectious Diseases, National Clinical Research Center for Infectious Diseases, The First Affiliated Hospital, School of Medicine, Zhejiang University, Hangzhou, China, ${ }^{2}$ Department of Geriatrics, Lishui Second People's Hospital, Lishui, China, ${ }^{3}$ Department of Intensive Care Unit, The First Affiliated Hospital, School of Medicine, Zhejiang University, Hangzhou, China, ${ }^{4}$ Institute of Hepatology and Metabolic Diseases, Hangzhou Normal University, Hangzhou, China,

${ }^{5}$ Institute of Translational Medicine, The Affiliated Hospital of Hangzhou Normal University, Hangzhou, China
\end{abstract}

Gut bacterial dysbiosis plays a vital role in the development of Alzheimer's disease (AD). However, our understanding of alterations to the gut fungal microbiota and their correlations with host immunity in $A D$ is still limited. Samples were obtained from 88 Chinese patients with $A D$, and 65 age- and gender-matched, cognitively normal controls. Using these samples, we investigated the fungal microbiota targeting internal transcribed spacer 2 (ITS2) rRNA genes using MiSeq sequencing, and analyzed their associations with the host immune response. Our data demonstrated unaltered fungal diversity but altered taxonomic composition of the fecal fungal microbiota in the $\mathrm{AD}$ patients. The analysis of the fungal microbiota was performed using $6,585,557$ high-quality reads $(2,932,482$ reads from the controls and 3,653,075 from the $A D$ patients), with an average of 43,042 reads per sample. We found that several key differential fungi such as Candida tropicalis and Schizophyllum commune were enriched in the AD patients, while Rhodotorula mucilaginosa decreased significantly. Interestingly, C. tropicalis and S. commune were positively correlated with IP-10 and TNF- $\alpha$ levels. In contrast, C. tropicalis was negatively correlated with IL-8 and IFN- $\gamma$ levels, and R. mucilaginosa was negatively correlated with TNF- $\alpha$ level. PiCRUSt analysis revealed that lipoic acid metabolism, starch and sucrose metabolism were significantly decreased in the AD fungal microbiota. This study is the first to demonstrate fecal fungal dysbiosis in stable AD patients at a deeper level, and to identify the key differential fungi involved in regulating host systemic immunity. The analysis of the fungal microbiota in AD performed here may provide novel insights into the etiopathogenesis of $A D$ and pave the way for improved diagnosis and treatment of AD.

Keywords: Alzheimer's disease, Candida, fungal microbiota, sequencing, TNF- $\alpha$

\section{INTRODUCTION}

Alzheimer's disease $(\mathrm{AD})$ is a neurodegenerative disorder characterized by a slow progression, which starts with mild memory loss and culminates in severe impairment of executive and cognitive functions. During the last decades, the prevalence of $\mathrm{AD}$ has been rapidly increasing due to the rise in life expectancy worldwide (Scheltens et al., 2016; Gaugler et al., 2019; Alzheimer's Association, 2020). It is estimated that by 2050, one in every 85 people will be living with AD (Brookmeyer et al., 2007). However, at present there are no mechanistic therapies or 
disease-modifying therapies available for AD (Honig et al., 2018). As a result, a diagnosis of $\mathrm{AD}$ has become one of the most devastating that patients and their families can receive. The financial burden imposed by $\mathrm{AD}$ is formidable due to the care needed by the growing number of patients with $\mathrm{AD}$ and other dementias. Given the clinical and financial burdens of the disease, $\mathrm{AD}$ should be regarded as a global public health priority.

Increasing evidence recognizes $\mathrm{AD}$ as a multifactorial and heterogeneous disease with multiple contributors to its pathophysiology, which is not restricted to effects on the central nervous system, but also includes strong interactions with external factors such as the gut microbiota. At autopsy, AD is characterized by amyloid-beta $(\mathrm{A} \beta)$ plaques and neurofibrillary tangles (LaFerla et al., 2007; Kinney et al., 2018; Sepulcre et al., 2018; Chen et al., 2020; Wan et al., 2020). Recently, a genome-wide spatial transcriptomic analysis identified an amyloid plaque-induced gene network, suggesting that $\mathrm{A} \beta$ plays active roles in the development of AD (Chen et al., 2020). In fact, in the last decades, the progress made by accounts of $\mathrm{AD}$ etiopathology focusing on the nervous system remains limited. Recent studies of the gut-brain axis have highlighted the potential roles of the gut microbiota in the development of various brain diseases, including $\mathrm{AD}$. Several studies have found an altered gut microbiota in $\mathrm{AD}$ patients, suggesting that the gut microbiota may be involved in $\mathrm{AD}$ pathogenesis (Vogt et al., 2017; Zhuang et al., 2018; Liu et al., 2019). Our group has also previously demonstrated that Clostridium butyricum and its metabolite butyrate can regulate the expression of $A \beta$, leading to an amelioration of cognitive deficits and neurodegeneration via modulation of the gut microbiota; these can therefore be considered to be potential psychobiotics (Sun et al., 2019a,b; Sun J. et al., 2020). Furthermore, a phase 3 clinical trial conducted in China by another group found that administering oligomannate led to a solid and consistent improvement in cognition in $\mathrm{AD}$ patients, suppressing gut dysbiosis and the associated phenylalanine/isoleucine accumulation, harnessing neuroinflammation, and reversing cognitive impairment (Wang et al., 2019). These findings indicate that gut dysbiosis can promote neuroinflammation during the progression of $\mathrm{AD}$, while restoration of the gut microbiota may be a novel strategy for treating $\mathrm{AD}$.

The gut microbiota is composed of a variety of microorganisms, including bacteria, viruses, fungi, and archaea. However, previous studies have mainly focused on the bacterial diversity and composition of the gut microbiota, while the fungal microbiota has not been explored extensively. Fungi are suggested to influence intestinal health and disease by suppressing the outgrowth of potential pathobionts, promoting immunoregulatory pathways, and modulating host metabolism (Huseyin et al., 2017; Ni et al., 2017; Sam et al., 2017; Chin et al., 2020). Several clinical studies have identified a distinct fungal microbiota dysbiosis in inflammatory bowel disease (IBD), primary sclerosing cholangitis, asthma, type 2 diabetes mellitus, chronic liver diseases, Parkinson's disease and other neurological diseases, and even colorectal cancer (Hoarau et al., 2016; Sokol et al., 2017; Forbes et al., 2018; Coker et al., 2019; Cirstea et al., 2020; Jayasudha et al., 2020; Jiang et al., 2020;
Lemoinne et al., 2020; Qiu et al., 2020; van Tilburg Bernardes et al., 2020; Ventin-Holmberg et al., 2020). Studies of animal models have found that commensal fungi can activate hostprotective immune pathways related to epithelial barrier integrity, but can also induce reactions that contribute to events associated with IBD (Iliev and Cadwell, 2020). In addition, by interacting with the bacteriome and/or virome, the gut fungal communities appears to be a cofactor in inflammation and in the host immune response, and therefore may contribute to various disease progression. Therefore, alterations to the fungal microbiota might actively contribute to the development of AD. In this study, we employed fungal-specific internal transcribed spacer (ITS) amplicon sequencing of a cross-sectional AD cohort to investigate associations between the fungal gut microbiota and $\mathrm{AD}$ using the $16 \mathrm{~S}$ rRNA high-throughput gene MiSeq platform. Furthermore, we performed correlation analysis between fungal taxa and clinical indicators to decipher their possible roles in the pathogenesis of $\mathrm{AD}$.

\section{MATERIALS AND METHODS}

\section{Subject Enrollment}

A total of 88 Chinese patients with well-controlled AD, whose diagnoses were based on the criteria of the National Institute of Neurological and Communicative Diseases and Stroke/AD and Related Disorders Association, were recruited from Lishui, Zhejiang province (China) from February 2019 to November 2019, along with 65 cognitively normal subjects as controls. The cognitive and functional status of each subject was scored using the Mini-Mental State Examination (MMSE, Chinese version), the current version in the Wechsler Adult Intelligence Scale series (WAIS-IV, published in 2008), and the Barthel Index of instrumental activities of daily living. Each participant was scanned using magnetic resonance imaging (MRI), with all $\mathrm{AD}$ patients diagnosed as showing brain atrophy. The detailed demographic data and medical history (including hypertension, diabetes mellitus, hypercholesterolemia, coronary heart disease, diarrhea, and constipation) were collected using a set of questionnaires. Exclusion criteria included: family history of dementia; any kind of other neurodegenerative disease such as Parkinson's disease; confirmed mental illness such as schizophrenia; any kind of tumor; antibiotic, prebiotic, probiotic, or synbiotic administration during the previous month; known active infections such as viral, bacterial, or fungal infections; and other diseases such as inflammatory bowel disease, irritable bowel syndrome, or other autoimmune diseases. The protocols for the study were approved by the Ethics Committee of Lishui Second People's Hospital (Zhejiang, China) and written informed consent was obtained from each of the subjects or their guardian before enrollment.

\section{Fecal Sample Collection and DNA Extraction}

Approximately $2 \mathrm{~g}$ of fresh fecal sample was collected from each subject in a sterile plastic cup, and stored at $-80^{\circ} \mathrm{C}$ after preparation within $15 \mathrm{~min}$, until its subsequent use. Metagenomic 
DNA was extracted from $300 \mathrm{mg}$ homogenized feces using a QIAamp DNA Stool Mini Kit (QIAGEN, Hilden, Germany) according to the manufacturer's instructions, with additional glass-bead beating steps performed using a Mini-Beadbeater (FastPrep; Thermo Electron, Boston, MA, United States). The amount of DNA was determined using a NanoDrop ND-1000 spectrophotometer (Thermo Electron). The integrity and size were verified by electrophoresis on a $1.0 \%$ agarose gel containing $0.5 \mathrm{mg} / \mathrm{ml}$ ethidium bromide. All DNA samples were stored at $-20^{\circ} \mathrm{C}$ prior to further analysis.

\section{Amplicon Library Construction and Sequencing}

Amplicon libraries were constructed using Illumina sequencingcompatible and barcode-indexed fungal PCR primers ITS3 (5'-GATGAAGAACGYAGYRAA-3') and ITS4 (5'-TCCTCCGC TTATTGATATGC- $3^{\prime}$ ), which target ITS2 rRNA genes (Degnan and Ochman, 2012). All PCR reactions were performed using HiFi HotStart ReadyMix (KAPA Biosystems) according to the manufacturer's protocol and approximately $50 \mathrm{ng}$ extracted DNA per reaction. Thermocycling conditions were set at $95^{\circ} \mathrm{C}$ for $1 \mathrm{~min}, 53^{\circ} \mathrm{C}$ for $1 \mathrm{~min}$, then $72^{\circ} \mathrm{C}$ for $1 \mathrm{~min}$ for 30 cycles, followed by a final extension at $72^{\circ} \mathrm{C}$ for $5 \mathrm{~min}$. All PCR reactions were performed in $50 \mu \mathrm{l}$ triplicates and combined after PCR. The amplicon library was prepared using a TruSeq DNA sample preparation kit (Illumina, San Diego, CA, United States). Prior to sequencing, the PCR products were extracted with the MinElute Gel Extraction Kit (QIAGEN) and quantified on a NanoDrop ND-1000 spectrophotometer (Thermo Electron) and a Qubit 2.0 Fluorometer (Invitrogen). The purified amplicons were then pooled in equimolar concentrations and the final concentration of the library was determined using the Qubit 2.0 Fluorometer. Negative DNA extraction samples (lysis buffer and kit reagents only) were amplified and sequenced as contamination controls. Sequencing was performed on a MiSeq instrument (Illumina) using a $300 \times 2$ V3 kit together with the PhiX Control V3 library (Illumina) (Ling et al., 2019; Wang et al., 2019). MiSeq sequencing and library construction were performed by technical staff at Hangzhou KaiTai Bio-lab.

\section{Bioinformatic Analysis}

The ITS sequence dataset generated by the MiSeq run were first merged and demultiplexed into per-sample data using QIIME version 1.9.0 with default parameters (Caporaso et al., 2010). Chimera sequences were detected and removed using USEARCH version 7 software based on the UCHIME algorithm (Edgar et al., 2011). The open-reference operational taxonomic unit (OTU) pick was then performed using USEARCH version 7 referenced against the Greengenes database version 13.8 at $97 \%$ sequence similarity (Edgar, 2010; McDonald et al., 2012). OTUs containing a number of sequences $<0.005 \%$ of the total number of sequences were discarded, as recommended (Navas-Molina et al., 2013). This resulted in an OTU table, which was used for subsequent downstream analysis.

To perform the taxonomic assignment, the most abundant sequence from each OTU was chosen as the representative sequences from that OTU. Taxonomic assignment of individual datasets was performed by classifying the data according to the UNITE database ${ }^{1}$ (Abarenkov et al., 2010). Alpha diversity was calculated based on the sequence similarity at the $97 \%$ level using QIIME software and Python scripts to calculate a range of estimators, including: index of observed species, abundancebased coverage estimator (ACE), Chaol estimator, Shannon, Simpson, evenness, and PD whole tree. Sequence coverage was assessed in Mothur software based on calculating rarefaction curves and Good's coverage (Good, 1953; Schloss et al., 2009). Beta diversity was estimated based on the Jaccard, BrayCurtis, unweighted UniFrac, and weighted UniFrac distances calculated with $10 \times$ subsampling in QIIME. These distances were visualized following principal coordinate analysis (PCoA) of the data (Lozupone and Knight, 2005). Hierarchical clustering was performed and a heatmap was generated using a customized script developed in the $\mathrm{R}$ statistical package, with Spearman's rank correlation coefficient as the distance measure. The output file was further analyzed using the Statistical Analysis of Metagenomic Profiles (STAMP) software package version 2.1.3 (Parks et al., 2014).

To perform the predictive functional analyses, PiCRUSt software version 1.0.0 was used to identify predicted gene families and associated pathways from the inferred metagenomes of taxa of interest identified during the compositional analyses; this analysis is based on the close link between phylogeny and function (Langille et al., 2013). Predicted functional genes were categorized based on the Clusters of Orthologous Groups (COG) database and on the Kyoto Encyclopedia of Genes and Genome (KEGG) orthology (KO), and then compared across patient groups using STAMP. Pathways and enzymes were assigned using the KEGG database options built into the pipeline. Pathways that were non-prokaryotic, had $<2$ sequences in each cohort, or had a difference in mean proportions $<0.1 \%$ were excluded from the analysis. The characterization of microorganismal features differentiating the gastric microbiota was performed using the linear discriminant analysis (LDA) effect size (LEfSe) method $^{2}$ for biomarker discovery, which emphasizes both statistical significance and biological relevance (Segata et al., 2011). Based on a normalized relative abundance matrix, the LEfSe method uses the Kruskal-Wallis rank sum test to detect features with significantly different abundances between assigned taxa and then performs LDA to estimate the effect size of each feature. A significant alpha threshold of 0.05 and an effect size threshold of 3 were used to identify all of the biomarkers discussed in this study.

\section{Systemic Inflammatory Cytokines Analysis}

Serum samples were obtained from the participants using their fasting blood in the early morning. The following cytokines were quantified using a 27-plex magnetic bead based immunoassay kit (Bio-Rad, Hercules, CA, United States): interleukin-1 $\beta$ (IL-1 $\beta$ ), IL-1 receptor antagonist (IL-1ra), IL-2, IL-4, IL-5,

\footnotetext{
${ }^{1}$ http://unite.ut.ee/repository.php

${ }^{2}$ http://huttenhower.sph.harvard.edu/galaxy/
} 
IL-6, IL-7, IL-8, IL-9, IL-10, IL-12 (p70), IL-13, IL-15, IL-17, eotaxin, fibroblast growth factor-basic (FGF-basic), granulocyte colony-stimulating factor (G-CSF), granulocyte-macrophage colony-stimulating factor (GM-CSF), interferon gamma (IFN- $\gamma$ ), interferon gamma-inducible protein 10 (IP-10), monocyte chemotactic protein-1 (MCP-1), macrophage inflammatory protein-1 $\alpha$ (MIP-1 $\alpha)$, platelet-derived growth factor (PDGF-bb), MIP-1 $\beta$, regulated upon activation normal T-cell expressed and secreted (RANTES), tumor necrosis factor-alpha (TNF- $\alpha$ ), and vascular endothelial growth factor (VEGF). The Bio-Plex 200 system (Bio-Rad) was used to analyze Bio-Rad 27-plex human group I cytokines, with the Bio-Plex assay performed according to the manufacturer's directions. The results were expressed as picograms per milliliter $(\mathrm{pg} / \mathrm{mL})$ using standard curves integrated into the assay and the Bio-Plex Manager v5.0 software (Bio-Rad), yielding reproducible intra-and inter-assay $\mathrm{CV}$ values of $5-8 \%$.

\section{Statistical Analysis}

White's nonparametric $t$-test, the independent $t$-test, or the Mann-Whitney $U$-test were applied to analyze continuous variables. Pearson's chi-square test or Fisher's exact test were used to analyze categorical variables between groups. Spearman's rank correlation test was used to perform correlation analyses. Statistical analysis was performed using SPSS version 19.0 (SPSS Inc., Chicago, IL, United States) and STAMP version 2.1.3 (Parks et al., 2014). R and GraphPad Prism v6.0 software were used to prepare graphs. All of the tests of significance performed were two sided, with $p<0.05$ or corrected $p<0.05$ considered statistically significant.

\section{Accession Number}

The sequence data from this study are deposited in the GenBank Sequence Read Archive with the accession number SRP292858.

\section{RESULTS}

\section{Subject Characteristics}

Eighty-eight stable AD patients and 65 age- and gender-matched cognitively normal, healthy controls were enrolled in the present study (Table 1). All participants were older than 65 years of age. There were no significant differences between the healthy controls and the $\mathrm{AD}$ patients in terms of gender, body mass index, drinking, or smoking, or in terms of comorbidities with hypertension, hypercholesterolemia, diabetes mellitus, or coronary heart disease (all ps > 0.05). However, MMSE, WAIS, and Barthel scores were clearly lower in AD patients than in the healthy controls (all ps $<0.05$ ).

\section{Unaltered Overall Structure of the Fungal Microbiota in Stable AD}

In the present study, 6,585,557 high-quality reads (2,932,482 reads from the controls and 3,653,075 from the $\mathrm{AD}$ patients) were obtained for subsequent analysis of the fungal microbiota, with an average of 43,042 reads per sample. Good's coverage
TABLE 1 | The fundamental information of subjects.

\begin{tabular}{|c|c|c|}
\hline Parameters & $\begin{array}{l}\text { AD patients } \\
\quad(n=88)\end{array}$ & $\begin{array}{l}\text { Healthy control } \\
\qquad(n=65)\end{array}$ \\
\hline Age $(y)$ & $74.28 \pm 8.89$ & $73.58 \pm 8.15$ \\
\hline Gender (male/female) & $40 / 48$ & $32 / 33$ \\
\hline $\mathrm{BMI}($ Mean \pm SD) & $23.20 \pm 3.25$ & $23.68 \pm 3.48$ \\
\hline Antibiotics use, no. & 0 & 0 \\
\hline \multicolumn{3}{|l|}{ Complications, no. } \\
\hline Hypertension & 30 & 19 \\
\hline Diabetes mellitus & 13 & 8 \\
\hline Hypercholesterolemia & 12 & 8 \\
\hline Coronary heart disease & 11 & 6 \\
\hline Diarrhea & 2 & 3 \\
\hline Constipation & 6 & 4 \\
\hline \multicolumn{3}{|c|}{ Cognitive and functional status } \\
\hline MMSE Score* & $4.35 \pm 5.89$ & $26.85 \pm 3.75$ \\
\hline WAIS Score ${ }^{\star}$ & $36.25 \pm 16.84$ & $91.25 \pm 11.28$ \\
\hline Barthel Score* & $22.38 \pm 24.20$ & $77.80 \pm 9.85$ \\
\hline
\end{tabular}

BMI, body mass index; SD, standard deviation; no., numbers; MMSE, Mini-Mental State Examination; WAIS, Wechsler Adult Intelligence Scale. ${ }^{*} p<0.05$.

was $99.99 \%$ in healthy controls and $99.98 \%$ in $\mathrm{AD}$ patients, respectively, suggesting that most of the fungal phylotypes (546 OTUs) in the AD-associated fungal microbiota were successfully identified. Interestingly, the calculated fungal alpha-diversity indices, including the Shannon and Simpson indices, show no significant changes of the $\mathrm{AD}$-associated fungal microbiota relative to that of the healthy controls (Figures 1A,B); however, there was a trend toward increasing fungal diversity in the stable $\mathrm{AD}$ patients. In terms of the richness indices, there were no significant changes in ACE, Chaol, and observed OTUs in stable $\mathrm{AD}$ patients compared with healthy controls (Figures 1C-E; all ps $>0.05$ ). Rarefaction plots reached a plateau for fungal species in both the $\mathrm{AD}$ and the control samples. We next assessed and compared the beta diversity of the fungal microbiota in $\mathrm{AD}$ patients to that in healthy controls based on the Bray-Curtis, Jaccard, unweighted UniFrac, and weighted UniFrac algorithms. The AD and control groups could not be divided into different clusters (Adonis test, $p>0.01$; Figures 1 F-I). In addition, the Venn diagram showed more unique phylotypes in $\mathrm{AD}$ patients than those in healthy controls (Figure 1J). Taken together, the alpha- and beta-diversity analyses demonstrate that the overall structure of the fungal microbiota in stable AD patients did not change obviously compared with that in the healthy controls.

\section{Taxonomic Alterations of Fecal Fungi in Stable AD}

The compositions of the fungal microbiota in the stable $\mathrm{AD}$ patients and healthy controls were assessed at different taxonomic levels (Figure 2). Overall, four phyla, 20 classes, 52 orders, 97 families, 148 genera, and 247 species were identified by this sequencing analysis. Among the fungal taxa, the phylum Ascomycota dominated the fungal microbiota, while Basidiomycota was observed as the 

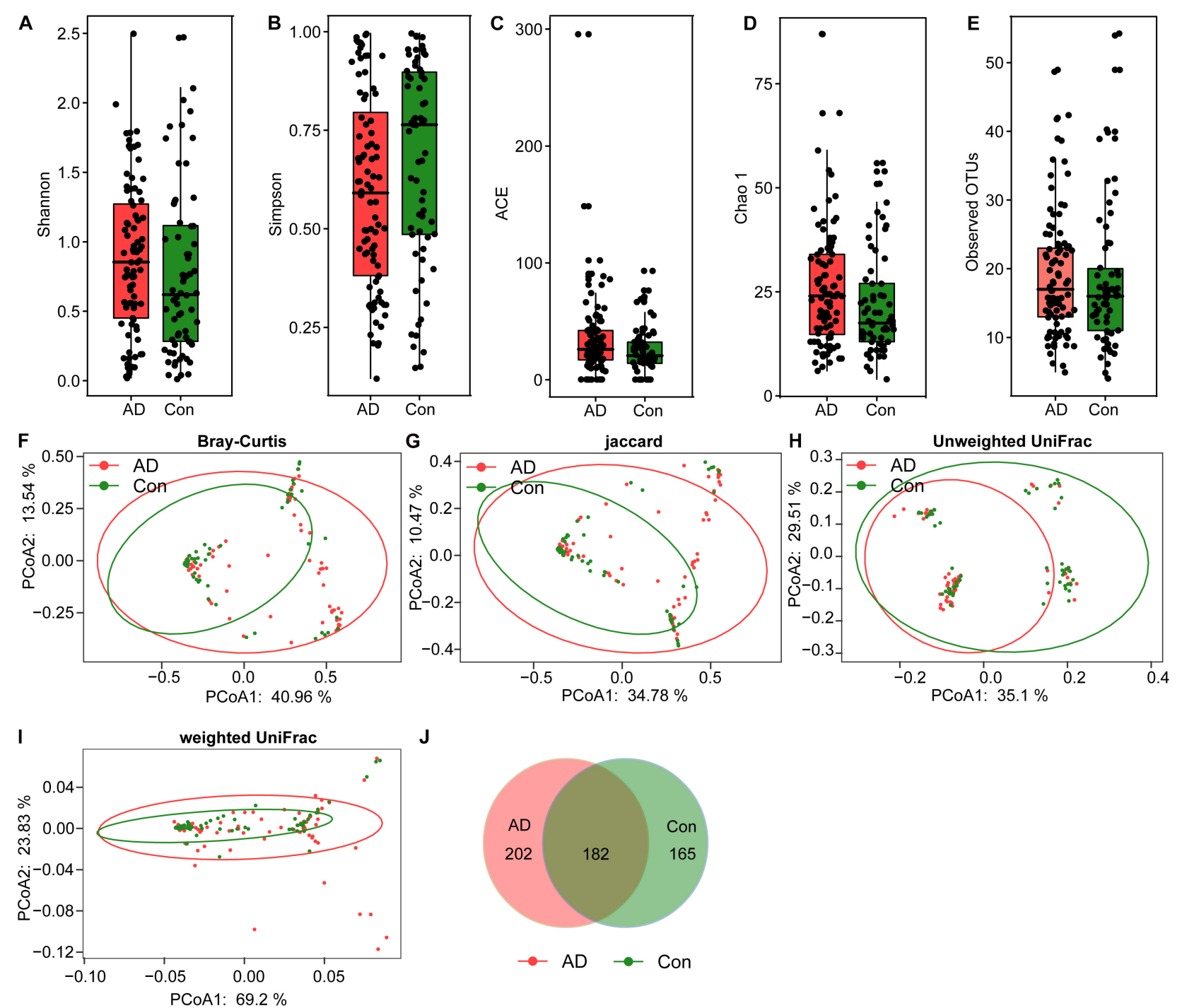

FIGURE 1 | Fungal diversity and richness of the fecal microbiota in Chinese AD patients. The diversity indices of Shannon (A) and Simpson (B), and the richness indices of the observed species (C), ACE (D), and Chao1 (E) were used to evaluate the overall structure of the fungal microbiota in the stable AD patients and the healthy controls. The data are presented as mean \pm standard deviation. Unpaired $t$-tests (two tailed) were used to analyze the variation between the groups. Principal coordinate analysis (PCoA) plots of individual fungal microbiota based on Bray-Curtis (F), Jaccard (G), and unweighted (H) and weighted (I) UniFrac distances in the AD patients and the healthy controls. Each symbol represents a sample. The Venn diagram illustrates the overlap of OTUs in the fungal microbiota between the two groups (J)

second most abundant phylum in both the $\mathrm{AD}$ and control groups. At the family level, Saccharomycetales family Incertae sedis, Trichocomaceae, Meruliaceae, Pleosporaceae, Trichosporonaceae, Schizophyllaceae, and Sclerotiniaceae were found to be dominant in the fungal microbiota, when unclassified fungal taxa were excluded. Interestingly, we found that a greater number of fungal families were observed in the $\mathrm{AD}$ patients compared with the healthy controls. At the genus level, classified genera including Candida, Aspergillus, Debaryomyces, Trichosporon, Wickerhamomyces, Schizophyllum, Phlebia, and Asterotremella were abundant in the fungal microbiota, both in the $\mathrm{AD}$ patients and the healthy controls. At the species level, fungal taxa including Candida albicans, Candida tropicalis,
Candida parapsilosis, Schizophyllum commune, Phlebia cf. subserialis MS42b, Asterotremella sp., Candida metapsilosis, and Wickerhamomyces anomalus were observed and classified. C. albicans, C. tropicalis, and C. parapsilosis were the most abundant species in the fungal microbiota, both in the $\mathrm{AD}$ patients and the healthy controls. Using the LEfSe method, discriminant analysis showed that many key taxa were clearly different between the $\mathrm{AD}$ and control groups (LDA score $>2$, $p<0.05$; Figure 3). Although the Basidiomycota/Ascomycota ratio is considered to be an indicator of fungal dysbiosis, we found no significant differences in these two abundant phyla between the $\mathrm{AD}$ patients and the healthy controls (Coker et al., 2019). The LEfSe analysis revealed that most of the differential 


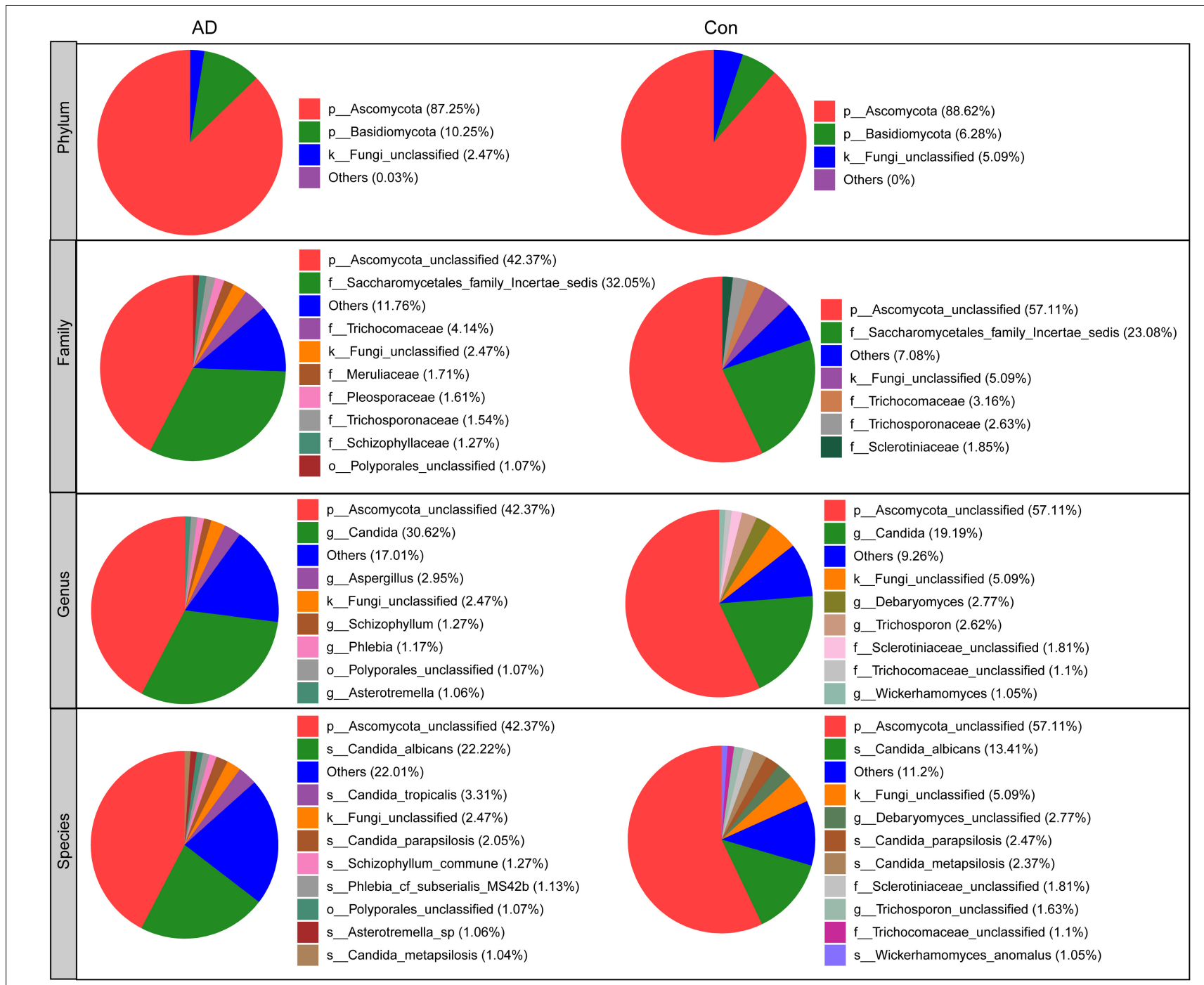

FIGURE 2 | Overall fungal microbiota composition in stable AD patients and healthy controls in phylum, family, genus, and species levels.

fungi could be classified at the species level. C. parapsilosis, Hannaella sp. CMON52, C. apicola, Cystofilobasidium capitatum, C. xylopsoci, C. zeylanoides, Malassezia globosa, Trichosporon veenhuisii, Bullera unica, Millerozyma farinosa, and Rhodotorula mucilaginosa were enriched in the healthy controls, while C. tropicalis, Trametes versicolor, S. commune, Davidiella tassiana, Exophiala dermatitidis, and Erythrobasidium hasegawianum were prevalent in stable $\mathrm{AD}$ patients. The abundances of the most numerous Candida species, such as C. albicans, did not show obvious changes between the two groups. Despite most of these differential species not being abundant, our results do nevertheless indicate fungal dysbiosis in stable $\mathrm{AD}$ patients.

\section{Fungal Functional Alterations in AD}

To identify metabolic and functional changes in the fungal microbiota between the AD patients and the controls, we used PiCRUSt to analyze the functional potential of the microbiota based on closed-reference OTU picking. We compared 105 KEGG pathways at level 3 and identified five KEGG categories with clearly differential abundances between the $\mathrm{AD}$ patients and the controls. The following KEGG categories decreased prominently in stable AD patients ( $p<0.05$; Figure 4): "lipoic acid metabolism," "cell cycle - Caulobacter," "starch and sucrose metabolism," "lysine degradation," and "phosphotransferase system (PTS)." These fungal functional alterations might participate in the pathogenesis and development of $\mathrm{AD}$.

\section{Correlations Between Key Differential Fungi and Host Immunity}

We found that the AD-associated clinical indicators we examined, including the MMSE, WAIS, and Barthel scores, were significantly lower in the stable $\mathrm{AD}$ patients compared with the healthy controls $(p<0.01)$. Using the Bio-Plex Pro human cytokine group I panel 27-plex analysis, we found 


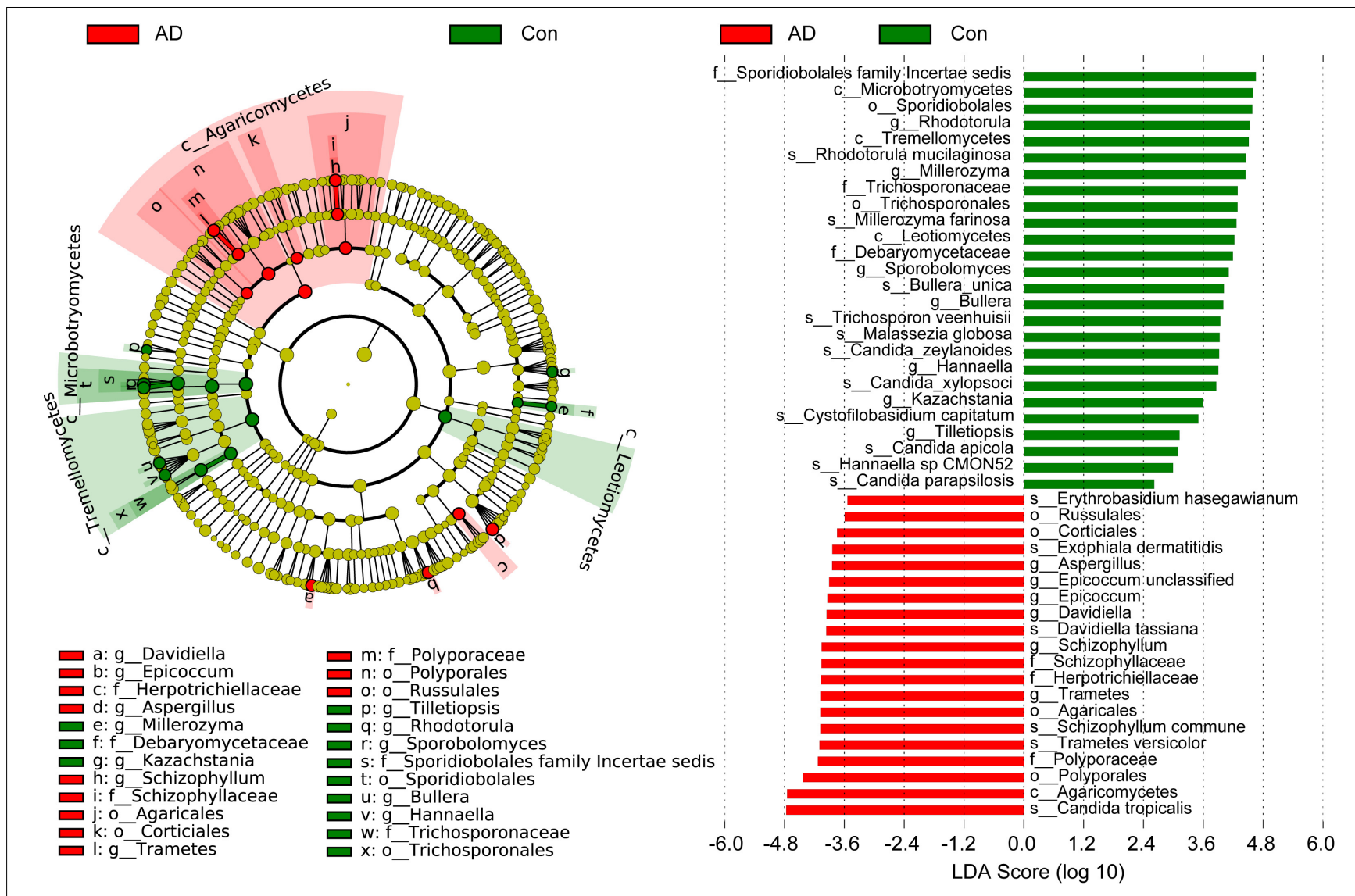

FIGURE 3 | Differential fungal taxa between the stable AD patients and the healthy controls. The LEfSe identified the taxa with the greatest differences in abundance between the AD patients and healthy controls. Only the taxa meeting a significant LDA threshold value of $>2$ are shown. These differential fungi could be used as potential biomarkers to distinguish AD patients from healthy controls.

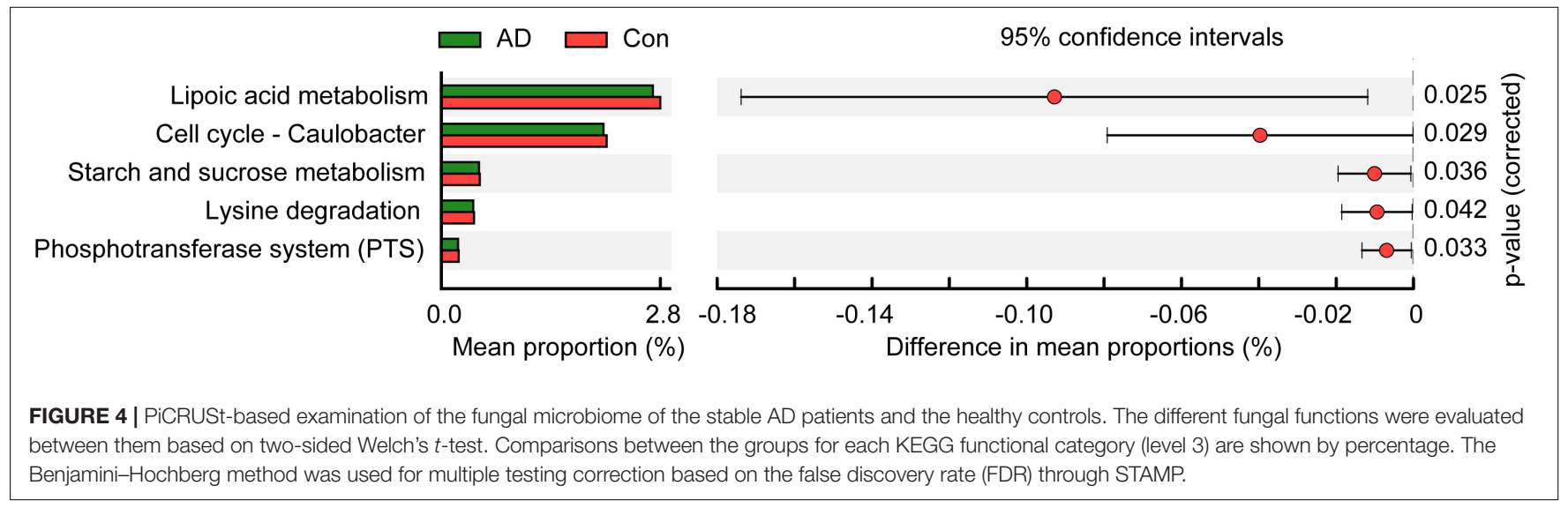

that, in the $\mathrm{AD}$ patients relative to the healthy controls, levels of anti-inflammatory cytokines (such as IFN- $\gamma$ ) and several chemokines (such as IL-8, MCP-1, and MIP-1a) were significantly lower $(p s<0.05)$, while levels of pro-inflammatory cytokines (such as TNF- $\alpha$ ) were markedly higher $(p s<0.05)$; furthermore, the level of IP-10 was also lower in the AD patients ( $p$ s < 0.05). Next, we investigated correlations between the key differential fungi and the altered cytokines using Spearman's rank correlation (Figure 5). We found that the enriched abundance of C. tropicalis in $\mathrm{AD}$ correlated negatively with levels of IL8 and IFN- $\gamma$, but correlated positively with levels of IP-10 and TNF- $\alpha$ ( $p$ s < 0.05). However, the abundance of C. parapsilosis, which was prevalent in healthy controls, was not correlated with altered cytokine levels. The abundance of another ADenriched fungi, $S$. commune, was positively correlated with levels of TNF- $\alpha$ and IP-10 ( $p$ s $<0.05$ ). The abundance of 

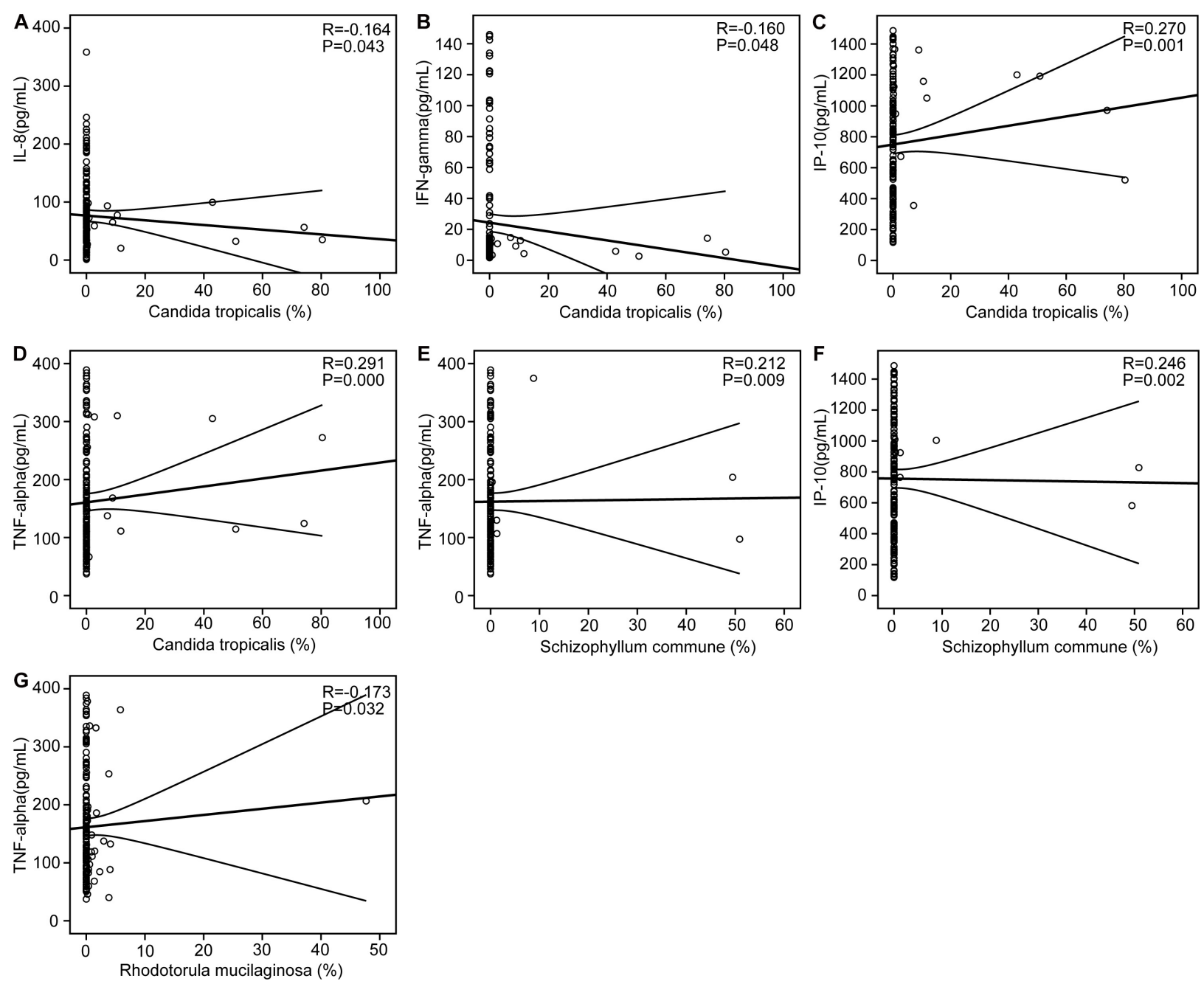

FIGURE 5 | Correlations between pro- and anti-inflammatory cytokines and chemokines with altered concentrations and the relative abundance of the key differential fungi. Correlation between the relative abundance of Candida tropicalis and the levels of IL-8 (A), IFN- $\gamma$ (B), IP-10 (C), and TNF- $\alpha$ (D); the relative abundance of Schizophyllum commune and the levels of TNF- $\alpha$ (E) and IP-10 (F); the relative abundance of Rhodotorula mucilaginosa and the levels of TNF- $\alpha$ (G). Spearman's rank correlation $(R)$ and probability $(P)$ were determined to evaluate the statistical importance.

R. mucilaginosa, a non-abundant fungi that was enriched in healthy controls, was negatively correlated with the level of TNF$\alpha(p<0.05)$. Thus, these results indicate that the altered key differential fungi regulated the systemic immune response in the $\mathrm{AD}$ patients, and may actively contribute to the development and progression of $\mathrm{AD}$.

\section{DISCUSSION}

In the present study, we observed fungal microbiota dysbiosis in Chinese patients with stable $\mathrm{AD}$ for the first time. Using highthroughput sequencing techniques, we found that alterations to several key differential fungi were associated with $\mathrm{AD}$, and showed clear correlations with the host immune response. These altered fungal taxa may play vital roles in the development and progression of AD.
The human gut microbiota is a complex and diverse ecosystem composed of bacteria, fungi, viruses, and archaea (Hamad et al., 2017). Of course, bacteria represent the majority of the microbial communities that inhabit the human gut, and their roles and mechanisms in human health and disease have been elucidated extensively. Maintaining a healthy balance of gut bacteria can promote good health, and indeed several previous studies have reported that $\mathrm{AD}$ pathology is closely correlated with alterations in gut bacterial profiles (Vogt et al., 2017; Zhuang et al., 2018; Li B.Y. et al., 2019; Liu et al., 2019). This suggests that human gut bacteria may play crucial roles in the etiopathology of $\mathrm{AD}$, such that these key differential bacteria could be used as potential targets for non-invasive diagnosis and treatment of AD. Unlike the bacterial community in the human gut, the composition and diversity of the fungal microbiota remains largely unexplored, because of the relatively low abundance of fungi in the human gut, combined 
with their neglect in research employing culture-based and molecular analyses (Marchesi, 2010). Nevertheless, intestinal fungi represent an important component of the microbiota in the human gastrointestinal tract that interacts with gut immune cells to maintain a healthy gut (Leonardi et al., 2018; Bacher et al., 2019). The gut fungal microbiota has recently been recognized as a novel and important player in the pathophysiology of intestinal and extraintestinal diseases (Huseyin et al., 2017), and is known to have a profound influence in modulating local as well as peripheral immune responses (Li X.V. et al., 2019). Because of the relatively low abundance of fungi in the human gastrointestinal tract (comprising only $2 \%$ of the human gut microbiota) (Anandakumar et al., 2019), little is known about their ability to influence human health and disease. With the advent of deep sequencing technologies, the composition and diversity of the fungal microbiota has been revealed, deepening and clarifying our understanding of the roles and mechanisms of intestinal fungi in host homeostasis (Iliev and Leonardi, 2017; Scheffold et al., 2020; Zhang et al., 2020; Zou et al., 2020). Recent studies have unveiled the potential roles that fungi play in modulating host immune homeostasis and inflammatory disease (Standaert-Vitse et al., 2006; Wheeler et al., 2016; Sokol et al., 2017; Yang et al., 2017; Cirstea et al., 2020; Huang et al., 2020; Jiang et al., 2020). However, the fungal microbiota is still a novel and emerging topic of research that continues to lag behind the level of research and understanding we have of the bacteriome. Prior to this study, there have not been any studies focusing on the roles and mechanisms of gut fungal communities in $\mathrm{AD}$, nor in any other neurodegenerative disorder. Nevertheless, the in-depth analysis of the $\mathrm{AD}$-associated fungal microbiota present here might provide novel insights into the development, progression, and treatment of $\mathrm{AD}$.

Fungal species detected in the human body mainly belong to three different phyla: Ascomycota, Basidiomycota, and Zygomycota (Gouba et al., 2013). Most fungal species can be considered commensal or mutualistic, while several yeast and filamentous fungi have been proved to be pathogenic (Parfrey et al., 2011). In the present study, we obtained more than 40,000 reads per sample, allowing us to characterize fungal diversity and composition in depth. In terms of the overall structure of the $\mathrm{AD}$-associated fungal microbiota, we found no significant differences in alpha- and beta-diversity in the stable $\mathrm{AD}$ patients compared with the healthy controls. The finding of a lack of alteration to the fungal diversity differs from the alterations to bacterial diversity observed in AD patients; however, the results of the fungal microbiota analysis presented here are consistent with those observed in Parkinson's disease (Cirstea et al., 2020). Other studies of the fungal microbiota in healthy adults have reported similar findings, including that the fungal microbiota is dominated by yeast and exhibits low fungal diversity and abundance, and high inter-individual variability (Nash et al., 2017; Auchtung et al., 2018). Liu et al. (2019) found that fecal microbial diversity decreased in $\mathrm{AD}$ patients compared with healthy controls. Given the significant inter-subject variability in our data, PCoA based on the Bray-Curtis, Jaccard, unweighted UniFrac, and weighted UniFrac algorithms was unable to divide the two groups into different clusters. In contrast, a prior study reported significant compositional differences in the intestinal bacteriome between $\mathrm{AD}$ patients and healthy controls based on PCoA using the Bray-Curtis dissimilarity (Liu et al., 2019). Thus, the unaltered diversity of the $\mathrm{AD}$-associated fungal microbiota observed in this study might imply that stable AD does not change the overall structure of the fungal microbiota.

In contrast to bacterial $16 \mathrm{~S}$ analyses, for which wellestablished, commonly accepted databases of sequences are available, fungal ITS analysis is relatively undeveloped (Tang et al., 2015), with the UNITE database (see text footnote 1) probably being the most commonly used fungal ITS database. Overall, we were able to classify our fungal ITS reads into different taxonomic levels, but for many of these reads, it was not possible to assign them into specific taxa based on the UNITE database. In the present study, we identified three phyla in the $\mathrm{AD}$-associated fungal microbiota, including Ascomycota, Basidiomycota, and Zygomycota, with most of sequences being assigned to the phylum Ascomycota. Recently, Coker et al. demonstrated that the Ascomycota/Basidiomycota ratio can be considered to be an indicator of fungal dysbiosis (Coker et al., 2019). However, in the present study, we found no significant difference in the Ascomycota/Basidiomycota ratio between the stable $\mathrm{AD}$ patients and the healthy controls. This may relate to the stable $\mathrm{AD}$ status of the patients, who were not receiving drug treatment. At the genus level, eight genera, namely Candida, Aspergillus, Debaryomyces, Trichosporon, Wickerhamomyces, Schizophyllum, Phlebia, and Asterotremella were the most abundant in the fecal fungal microbiota and exhibited different levels of prevalence in the $\mathrm{AD}$ patients compared with the healthy controls. Specifically, we found that Aspergillus, Schizophyllum, and Epicoccum were enriched in the AD patients, while Rhodotorula, Millerozyma, Sporobolomyces, Bullera, Hannaella, Kazachstania, and Tilletiopsis were more prevalent in the healthy controls. These alterations to fungal microbiota composition suggest fungal dysbiosis in the $\mathrm{AD}$-associated fungal microbiota. Furthermore, LEfSe analysis identified several AD-enriched fungal species, including C. tropicalis, Trametes versicolor, S. commune, Davidiella tassiana, Exophiala dermatitidis, and Erythrobasidium hasegawianum, which belong to the genera mentioned above. In contrast, other species, such as C. parapsilosis, Hannaella sp. CMON52, C. apicola, and R. mucilaginosa, were prevalent in the healthy controls. Taken together, these species could be used as potential biomarkers for the non-invasive diagnosis of $\mathrm{AD}$. As with bacteria, fungi can be beneficial to host immunity, but they can also exert deleterious effects under pathological conditions associated with disease. Among the altered fungal species identified in the present study, C. tropicalis, which is one of the most abundant pathogenic species in the central nervous system (Sanches et al., 2019), was found to be increased significantly in AD patients. Furthermore, we found that the abundance of $C$. tropicalis was negatively correlated with levels of IL- 8 and IFN- $\gamma$, and positively correlated with those of IP-10 and TNF- $\alpha$. Our data suggest that $C$. tropicalis might participate in actively regulating the host systemic immune response. Similarly, S. commune, a sap-rot Basidiomycota and cosmopolitan species, also exhibited immunomodulatory properties. As with C. tropicalis, we found that the enriched 
abundance of $S$. commune in $\mathrm{AD}$ patients correlated positively with levels of IP-10 and TNF- $\alpha$. In contrast, the abundance of another species, $R$. mucilaginosa, was reduced significantly in the $\mathrm{AD}$-associated fungal microbiota. Our correlation analysis found that the abundance of $R$. mucilaginosa correlated negatively with the level of TNF- $\alpha$. In addition, the inferred function of the fungal microbiota also changed significantly in the $\mathrm{AD}$ patients. Five KEGG pathways were significantly decreased in AD patients, namely: "lipoic acid metabolism," "cell cycle - Caulobacter," "starch and sucrose metabolism," "lysine degradation," and "phosphotransferase system (PTS)." Previous studies have demonstrated that lipoic acid can function as a novel anti-inflammatory and neuroprotective treatment for $\mathrm{AD}$ and related dementias (Holmquist et al., 2007; Maczurek et al., 2008; Sancheti et al., 2014). Sun C. et al. (2020) found that the biological pathway "starch and sucrose metabolism" was associated with serum metabolomic biomarkers that were able to distinguish $\mathrm{AD}$ patients from healthy controls. Of course, the relationships between alterations to key functional fungi (especially nonabundant fungi) or inferred functions and $\mathrm{AD}$ are still unclear. Collectively, alterations to the composition of the fungal gut microbiota, especially to key functional fungi, and changes in inferred functions actively participate in the development and progression of $\mathrm{AD}$ by regulating the host immune response and modulating host metabolic processes.

This study of the fungal microbiota in $\mathrm{AD}$ is the first to be conducted, but it did have several limitations. Firstly, the fungal ITS sequencing-based community analysis targeted ITS3/ITS4 with specific PCR primers, which successfully enabled ITS sequencing reads with an average length of nearly 350 bp. However, many ITS sequences could not be correctly annotated and were instead simply annotated as "fungi," which affected the subsequent analyses of these data. Longer ITS sequences, metagenomic sequences, or the use of well-established fungal databases might help to improve taxonomic assignment. Secondly, most of the fungi identified belonged to non-abundant fungal taxa (with low relative abundance) and could not be detected in all samples, exhibiting a low detection rate. The significant inter-subject variations might have influenced the identification of clinically important fungal species. Thirdly, contamination from food could not be completely excluded in the process of collecting feces; thus, several of the fungal species identified might be associated with the food supplement. Fourthly, AD patients with a newly diagnosed onset were not enrolled in our study. Including these patients in the study might have allowed changes in the abundance patterns of the fungal microbiota to be understood more clearly.

In summary, the present study was the first to analyze the fungal microbiota in AD patients. Although fecal fungal diversity did not change significantly between the $\mathrm{AD}$ patients and the healthy controls, the composition of the fungal microbiota was significantly altered. Several key fungal species, including

\section{REFERENCES}

Abarenkov, K., Henrik Nilsson, R., Larsson, K. H., Alexander, I. J., Eberhardt, U., Erland, S., et al. (2010). The UNITE database for molecular identification
C. tropicalis, Trametes versicolor, S. commune, Davidiella tassiana, Exophiala dermatitidis, Erythrobasidium hasegawianum, were enriched in the $\mathrm{AD}$-associated fungal microbiota, while abundances of $C$. parapsilosis, Hannaella sp. CMON52, C. apicola, and R. mucilaginosa clearly decreased. Key functional fungi, such as C. tropicalis, S. commune, and R. mucilaginosa, were shown to actively participate in regulating the host systemic immune response. The large, case-control study presented here provides novel insights in the etiopathogenesis of $\mathrm{AD}$ and paves the way for improved diagnosis and treatment of $\mathrm{AD}$ in the future.

\section{DATA AVAILABILITY STATEMENT}

The original contributions presented in the study are publicly available. This data can be found here: GenBank Sequence Read Archive with the accession number SRP292858.

\section{ETHICS STATEMENT}

The studies involving human participants were reviewed and approved by the Ethics Committee of Lishui Second People's Hospital (Zhejiang, China). The patients/participants provided their written informed consent to participate in this study.

\section{AUTHOR CONTRIBUTIONS}

ZL, SW, and MZ conceived and designed the experiments. ZL, $\mathrm{XY}, \mathrm{YC}, \mathrm{LS}, \mathrm{XL}, \mathrm{RJ}$, and $\mathrm{MZ}$ performed the experiments. ZL, XY, LS, and MZ analyzed the data. ZL, XL, and LS wrote and edited the manuscript. All authors read and approved the final manuscript.

\section{FUNDING}

This work was funded by the grants of the National Natural Science Foundation of China (81771724, 31700800, and 81790631), National S\&T Major Project of China (2018YFC2000500), S\&T Major Project of Lishui (2017YSKZ-01 and 2017ZDYF04), Lishui \& ZJU Cooperation Project (2018zdhz07), and Foundation of China's State Key Laboratory for Diagnosis and Treatment of Infectious Diseases.

\section{ACKNOWLEDGMENTS}

The authors thank all of the participants who recruited patients in this study.

of fungi-recent updates and future perspectives. New Phytol. 186, 281-285 doi: 10.1111/j.1469-8137.2009.03160.x

Alzheimer's Association (2020). 2020 Alzheimer's disease facts and figures. Alzheimers Dement. 16, 391-460. doi: 10.1002/alz.12068 
Anandakumar, A., Pellino, G., Tekkis, P., and Kontovounisios, C. (2019). Fungal microbiome in colorectal cancer: a systematic review. Updates Surg. 71, 625630. doi: 10.1007/s13304-019-00683-8

Auchtung, T. A., Fofanova, T. Y., Stewart, C. J., Nash, A. K., Wong, M. C., Gesell, J. R., et al. (2018). Investigating colonization of the healthy adult gastrointestinal tract by fungi. $m$ Sphere 3:e00092-18. doi: 10.1128/mSphere.00092-18

Bacher, P., Hohnstein, T., Beerbaum, E., Röcker, M., Blango, M. G., Kaufmann, S., et al. (2019). Human Anti-fungal Th17 immunity and pathology rely on crossreactivity against Candida albicans. Cell 176, 1340.e15-1355.e15. doi: 10.1016/j. cell.2019.01.041

Brookmeyer, R., Johnson, E., Ziegler-Graham, K., and Arrighi, H. M. (2007). Forecasting the global burden of Alzheimer's disease. Alzheimers Dement. 3, 186-191. doi: 10.1016/j.jalz.2007.04.381

Caporaso, J. G., Kuczynski, J., Stombaugh, J., Bittinger, K., Bushman, F. D., Costello, E. K., et al. (2010). QIIME allows analysis of high-throughput community sequencing data. Nat. Methods 7, 335-336. doi: 10.1038/nmeth. f.303

Chen, W. T., Lu, A., Craessaerts, K., Pavie, B., Sala Frigerio, C., Corthout, N., et al. (2020). Spatial transcriptomics and in situ sequencing to study Alzheimer's Disease. Cell 182, 976.e19-991.e19. doi: 10.1016/j.cell.2020.06.038

Chin, V. K., Yong, V. C., Chong, P. P., Amin Nordin, S., Basir, R., and Abdullah, M. (2020). Mycobiome in the gut: a multiperspective review. Mediators Inflamm. 2020:9560684. doi: 10.1155/2020/9560684

Cirstea, M. S., Sundvick, K., Golz, E., Yu, A. C., Boutin, R. C. T., Kliger, D., et al. (2020). The gut mycobiome in Parkinson's disease. J. Parkinsons Dis. (in press). doi: $10.3233 /$ jpd-202237

Coker, O. O., Nakatsu, G., Dai, R. Z., Wu, W. K. K., Wong, S. H., Ng, S. C., et al. (2019). Enteric fungal microbiota dysbiosis and ecological alterations in colorectal cancer. Gut 68, 654-662. doi: 10.1136/gutjnl-2018-317178

Degnan, P. H., and Ochman, H. (2012). Illumina-based analysis of microbial community diversity. ISME J. 6, 183-194. doi: 10.1038/ismej.2011.74

Edgar, R. C. (2010). Search and clustering orders of magnitude faster than BLAST. Bioinformatics 26, 2460-2461. doi: 10.1093/bioinformatics/btq461

Edgar, R. C., Haas, B. J., Clemente, J. C., Quince, C., and Knight, R. (2011). UCHIME improves sensitivity and speed of chimera detection. Bioinformatics 27, 2194-2200. doi: 10.1093/bioinformatics/btr381

Forbes, J. D., Bernstein, C. N., Tremlett, H., Van Domselaar, G., and Knox, N. C. (2018). A Fungal world: could the gut mycobiome be involved in neurological disease? Front. Microbiol. 9:3249. doi: 10.3389/fmicb.2018.03249

Gaugler, J., James, B., Johnson, T., Marin, A., Weuve, J., and Alzheimer's disease (2019). 2019 Alzheimer's disease facts and figures. Alzheimers Dement. 15, 321-387. doi: 10.1016/j.jalz.2019.01.010

Good, I. J. (1953). The population frequencies of species and the estimation of population parameters. Biometrika 40, 237-264. doi: 10.2307/2333344

Gouba, N., Raoult, D., and Drancourt, M. (2013). Plant and fungal diversity in gut microbiota as revealed by molecular and culture investigations. PLoS One 8:e59474. doi: 10.1371/journal.pone.0059474

Hamad, I., Ranque, S., Azhar, E. I., Yasir, M., Jiman-Fatani, A. A., Tissot-Dupont, H., et al. (2017). Culturomics and amplicon-based metagenomic approaches for the study of fungal population in human gut microbiota. Sci. Rep. 7:16788. doi: 10.1038/s41598-017-17132-4

Hoarau, G., Mukherjee, P. K., Gower-Rousseau, C., Hager, C., Chandra, J., Retuerto, M. A., et al. (2016). Bacteriome and mycobiome interactions underscore microbial dysbiosis in familial Crohn's Disease. mBio 7:e01250-16. doi: 10.1128/mBio.01250-16

Holmquist, L., Stuchbury, G., Berbaum, K., Muscat, S., Young, S., Hager, K., et al. (2007). Lipoic acid as a novel treatment for Alzheimer's disease and related dementias. Pharmacol. Ther. 113, 154-164. doi: 10.1016/j.pharmthera.2006. 07.001

Honig, L. S., Vellas, B., Woodward, M., Boada, M., Bullock, R., Borrie, M., et al. (2018). Trial of solanezumab for mild dementia due to Alzheimer's Disease. N. Engl. J. Med. 378, 321-330. doi: 10.1056/NEJMoa1705971

Huang, C., Yu, Y., Du, W., Liu, Y., Dai, R., Tang, W., et al. (2020). Fungal and bacterial microbiome dysbiosis and imbalance of trans-kingdom network in asthma. Clin. Transl. Allergy. 10:42. doi: 10.1186/s13601-020-00345-8

Huseyin, C. E., O’Toole, P. W., Cotter, P. D., and Scanlan, P. D. (2017). Forgotten fungi-the gut mycobiome in human health and disease. FEMS Microbiol. Rev. 41, 479-511. doi: 10.1093/femsre/fuw047
Iliev, I. D., and Cadwell, K. (2020). Effects of intestinal fungi and viruses on immune responses and inflammatory bowel diseases. Gastroenterology S00165085, 35564-35565. doi: 10.1053/j.gastro.2020.06.100

Iliev, I. D., and Leonardi, I. (2017). Fungal dysbiosis: immunity and interactions at mucosal barriers. Nat. Rev. Immunol. 17, 635-646. doi: 10.1038/nri.2017.55

Jayasudha, R., Das, T., Kalyana Chakravarthy, S., Sai Prashanthi, G., Bhargava, A., Tyagi, M., et al. (2020). Gut mycobiomes are altered in people with type 2 diabetes mellitus and diabetic retinopathy. PLoS One 15:e0243077. doi: 10.1371/ journal.pone.0243077

Jiang, L., Stärkel, P., Fan, J. G., Fouts, D. E., Bacher, P., and Schnabl, B. (2020). The gut mycobiome: a novel player in chronic liver diseases. J. Gastroenterol. (in press). doi: 10.1007/s00535-020-01740-5

Kinney, J. W., Bemiller, S. M., Murtishaw, A. S., Leisgang, A. M., Salazar, A. M., and Lamb, B. T. (2018). Inflammation as a central mechanism in Alzheimer's disease. Alzheimers Dement. 4, 575-590. doi: 10.1016/j.trci.2018.06.014

LaFerla, F. M., Green, K. N., and Oddo, S. (2007). Intracellular amyloid-beta in Alzheimer's disease. Nat. Rev. Neurosci. 8, 499-509. doi: 10.1038/nrn2168

Langille, M. G., Zaneveld, J., Caporaso, J. G., McDonald, D., Knights, D., Reyes, J. A., et al. (2013). Predictive functional profiling of microbial communities using 16S rRNA marker gene sequences. Nat. Biotechnol. 31, 814-821. doi: $10.1038 /$ nbt. 2676

Lemoinne, S., Kemgang, A., Ben Belkacem, K., Straube, M., Jegou, S., Corpechot, C., et al. (2020). Fungi participate in the dysbiosis of gut microbiota in patients with primary sclerosing cholangitis. Gut 69, 92-102. doi: 10.1136/gutjnl-2018317791

Leonardi, I., Li, X., Semon, A., Li, D., Doron, I., Putzel, G., et al. (2018). CX3CR1(+) mononuclear phagocytes control immunity to intestinal fungi. Science 359, 232-236. doi: 10.1126/science.aao1503

Li, B. Y., He, Y. X., Ma, J. F., Huang, P., Du, J. J., Cao, L., et al. (2019). Mild cognitive impairment has similar alterations as Alzheimer's disease in gut microbiota. Alzheimers Dement. 15, 1357-1366. doi: 10.1016/j.jalz.2019.07.002

Li, X. V., Leonardi, I., and Iliev, I. D. (2019). Gut Mycobiota in Immunity and Inflammatory Disease. Immunity 50, 1365-1379. doi: 10.1016/j.immuni.2019. 05.023

Ling, Z., Shao, L., Liu, X., Cheng, Y., Yan, C., Mei, Y., et al. (2019). Regulatory T cells and plasmacytoid dendritic cells within the tumor microenvironment in gastric cancer are correlated with gastric microbiota dysbiosis: a preliminary study. Front. Immunol. 10:533. doi: 10.3389/fimmu.2019.00533

Liu, P., Wu, L., Peng, G., Han, Y., Tang, R., Ge, J., et al. (2019). Altered microbiomes distinguish Alzheimer's disease from amnestic mild cognitive impairment and health in a Chinese cohort. Brain Behav. Immun. 80, 633-643. doi: 10.1016/j. bbi.2019.05.008

Lozupone, C., and Knight, R. (2005). UniFrac: a new phylogenetic method for comparing microbial communities. Appl. Environ. Microbiol. 71, 8228-8235. doi: 10.1128/AEM.71.12.8228-8235.2005

Maczurek, A., Hager, K., Kenklies, M., Sharman, M., Martins, R., Engel, J., et al. (2008). Lipoic acid as an anti-inflammatory and neuroprotective treatment for Alzheimer's disease. Adv. Drug Deliv. Rev. 60, 1463-1470. doi: 10.1016/j.addr. 2008.04.015

Marchesi, J. R. (2010). Prokaryotic and eukaryotic diversity of the human gut. $A d v$. Appl. Microbiol. 72, 43-62. doi: 10.1016/s0065-2164(10)72002-5

McDonald, D., Price, M. N., Goodrich, J., Nawrocki, E. P., DeSantis, T. Z., Probst, A., et al. (2012). An improved Greengenes taxonomy with explicit ranks for ecological and evolutionary analyses of bacteria and archaea. ISME J. 6, 610-618. doi: 10.1038/ismej.2011.139

Nash, A. K., Auchtung, T. A., Wong, M. C., Smith, D. P., Gesell, J. R., Ross, M. C., et al. (2017). The gut mycobiome of the human microbiome project healthy cohort. Microbiome 5:153. doi: 10.1186/s40168-017-0373-4

Navas-Molina, J. A., Peralta-Sanchez, J. M., Gonzalez, A., McMurdie, P. J., Vazquez-Baeza, Y., Xu, Z. J., et al. (2013). Advancing our understanding of the human microbiome using QIIME. Method Enzymol. 531, 371-444. doi: 10.1016/B978-0-12-407863-5.00019-8

Ni, J., Wu, G. D., Albenberg, L., and Tomov, V. T. (2017). Gut microbiota and IBD: causation or correlation? Nat. Rev. Gastroenterol. Hepatol. 14, 573-584. doi: $10.1038 /$ nrgastro.2017.88

Parfrey, L. W., Walters, W. A., and Knight, R. (2011). Microbial eukaryotes in the human microbiome: ecology, evolution, and future directions. Front. Microbiol. 2:153. doi: $10.3389 /$ fmicb. 2011.00153 
Parks, D. H., Tyson, G. W., Hugenholtz, P., and Beiko, R. G. (2014). STAMP: statistical analysis of taxonomic and functional profiles. Bioinformatics 30, 3123-3124. doi: 10.1093/bioinformatics/btu494

Qiu, X., Zhao, X., Cui, X., Mao, X., Tang, N., Jiao, C., et al. (2020). Characterization of fungal and bacterial dysbiosis in young adult Chinese patients with Crohn's disease. Therap. Adv. Gastroenterol. 13:1756284820971202. doi: 10. $1177 / 1756284820971202$

Sam, Q. H., Chang, M. W., and Chai, L. Y. (2017). The fungal mycobiome and its interaction with gut bacteria in the host. Int. J. Mol. Sci. 18:330. doi: 10.3390/ ijms 18020330

Sanches, M. D., Mimura, L. A. N., Oliveira, L. R. C., Ishikawa, L. L. W., Garces, H. G., Bagagli, E., et al. (2019). Differential behavior of non-albicans Candida species in the central nervous system of immunocompetent and immunosuppressed mice. Front. Microbiol. 9:2968. doi: 10.3389/fmicb.2018. 02968

Sancheti, H., Kanamori, K., Patil, I., Díaz Brinton, R., Ross, B. D., and Cadenas, E. (2014). Reversal of metabolic deficits by lipoic acid in a triple transgenic mouse model of Alzheimer's disease: a 13C NMR study. J. Cereb. Blood Flow Metab. 34, 288-296. doi: 10.1038/jcbfm.2013.196

Scheffold, A., Bacher, P., and LeibundGut-Landmann, S. (2020). T cell immunity to commensal fungi. Curr. Opin Microbiol. 58, 116-123. doi: 10.1016/j.mib.2020. 09.008

Scheltens, P., Blennow, K., Breteler, M. M., de Strooper, B., Frisoni, G. B., Salloway, S., et al. (2016). Alzheimer's disease. Lancet 388, 505-517. doi: 10.1016/s01406736(15)01124- 1

Schloss, P. D., Westcott, S. L., Ryabin, T., Hall, J. R., Hartmann, M., Hollister, E. B., et al. (2009). Introducing mothur: open-source, platform-independent, community-supported software for describing and comparing microbial communities. Appl. Environ. Microbiol. 75, 7537-7541. doi: 10.1128/Aem. 01541-09

Segata, N., Izard, J., Waldron, L., Gevers, D., Miropolsky, L., Garrett, W. S., et al. (2011). Metagenomic biomarker discovery and explanation. Genome Biol. 12:R60. doi: 10.1186/gb-2011-12-6-r60

Sepulcre, J., Grothe, M. J., d'Oleire Uquillas, F., Ortiz-Terán, L., Diez, I., Yang, H. S., et al. (2018). Neurogenetic contributions to amyloid beta and tau spreading in the human cortex. Nat. Med. 24, 1910-1918. doi: 10.1038/s41591-018-0206-4

Sokol, H., Leducq, V., Aschard, H., Pham, H. P., Jegou, S., Landman, C., et al. (2017). Fungal microbiota dysbiosis in IBD. Gut 66, 1039-1048. doi: 10.1136/ gutjnl-2015-310746

Standaert-Vitse, A., Jouault, T., Vandewalle, P., Mille, C., Seddik, M., Sendid, B., et al. (2006). Candida albicans is an immunogen for anti-Saccharomyces cerevisiae antibody markers of Crohn's disease. Gastroenterology 130, 17641775. doi: 10.1053/j.gastro.2006.02.009

Sun, C., Gao, M., Wang, F., Yun, Y., Sun, Q., Guo, R., et al. (2020). Serum metabolomic profiling in patients with Alzheimer disease and amnestic mild cognitive impairment by GC/MS. Biomed. Chromatogr. 34:e4875. doi: 10.1002/ bmc. 4875

Sun, J., Xu, J., Yang, B., Chen, K., Kong, Y., Fang, N., et al. (2020). Effect of clostridium butyricum against microglia-mediated neuroinflammation in Alzheimer's Disease via regulating gut microbiota and metabolites butyrate. Mol. Nutr. Food Res. 64:e1900636. doi: 10.1002/mnfr.201900636

Sun, J., Liu, S. Z., Ling, Z. X., Wang, F. Y., Ling, Y., Gong, T. Y., et al. (2019a). Fructooligosaccharides ameliorating cognitive deficits and neurodegeneration in APP/PS1 Transgenic mice through modulating gut microbiota. J. Agrc. Food Chem. 67, 3006-3017. doi: 10.1021/acs.jafc.8b07313
Sun, J., Xu, J., Ling, Y., Wang, F., Gong, T., Yang, C., et al. (2019b). Fecal microbiota transplantation alleviated Alzheimer's disease-like pathogenesis in APP/PS1 transgenic mice. Transl. Psychiatry 9:189. doi: 10.1038/s41398-0190525-3

Tang, J., Iliev, I. D., Brown, J., Underhill, D. M., and Funari, V. A. (2015). Mycobiome: approaches to analysis of intestinal fungi. J. Immunol. Methods 421, 112-121. doi: 10.1016/j.jim.2015.04.004

van Tilburg Bernardes, E., Gutierrez, M. W., and Arrieta, M. C. (2020). The fungal microbiome and asthma. Front. Cell Infect. Microbiol. 10:583418. doi: 10.3389/ fcimb. 2020.583418

Ventin-Holmberg, R., Eberl, A., Saqib, S., Korpela, K., Virtanen, S., Sipponen, T., et al. (2020). Bacterial and fungal profiles as markers of infliximab drug response in inflammatory bowel disease. J. Crohns Colitis jjaa252. (in press). doi: 10.1093/ecco-jcc/jjaa252

Vogt, N. M., Kerby, R. L., Dill-McFarland, K. A., Harding, S. J., Merluzzi, A. P., Johnson, S. C., et al. (2017). Gut microbiome alterations in Alzheimer's disease. Sci. Rep. U. K. 7:13537. doi: 10.1038/S41598-017-13 601-Y

Wan, Y. W., Al-Ouran, R., Mangleburg, C. G., Perumal, T. M., Lee, T. V., Allison, K., et al. (2020). Meta-analysis of the Alzheimer's disease human brain transcriptome and functional dissection in mouse models. Cell Rep. 32:107908. doi: 10.1016/j.celrep.2020.107908

Wang, X., Sun, G., Feng, T., Zhang, J., Huang, X., Wang, T., et al. (2019). Sodium oligomannate therapeutically remodels gut microbiota and suppresses gut bacterial amino acids-shaped neuroinflammation to inhibit Alzheimer's disease progression. Cell Res. 29, 787-803. doi: 10.1038/s41422-019-0216-x

Wheeler, M. L., Limon, J. J., Bar, A. S., Leal, C. A., Gargus, M., Tang, J., et al. (2016). Immunological consequences of intestinal fungal dysbiosis. Cell Host Microbe 19, 865-873. doi: 10.1016/j.chom.2016.05.003

Yang, A. M., Inamine, T., Hochrath, K., Chen, P., Wang, L., Llorente, C., et al. (2017). Intestinal fungi contribute to development of alcoholic liver disease. J. Clin. Invest. 127, 2829-2841. doi: 10.1172/jci90562

Zhang, C. B., Ren, C. H., Wang, Y. L., Wang, Q. Q., Wang, Y. S., and Weng, Q. B. (2020). Uncovering fungal community composition in natural habitat of Ophiocordyceps sinensis using high-throughput sequencing and culturedependent approaches. BMC Microbiol. 20:331. doi: 10.1186/s12866-02001994-2

Zhuang, Z. Q., Shen, L. L., Li, W. W., Fu, X., Zeng, F., Gui, L., et al. (2018). Gut microbiota is altered in patients with Alzheimer's Disease. J. Alzheimers Dis. 63, 1337-1346. doi: 10.3233/Jad-180176

Zou, R., Wang, Y., Duan, M., Guo, M., Zhang, Q., and Zheng, H. (2020). Dysbiosis of gut fungal microbiota in children with autism spectrum disorders. J. Autism. Dev. Disord. (in press). doi: 10.1007/s10803-020-04543-y

Conflict of Interest: The authors declare that the research was conducted in the absence of any commercial or financial relationships that could be construed as a potential conflict of interest.

Copyright (c) 2021 Ling, Zhu, Liu, Shao, Cheng, Yan, Jiang and Wu. This is an open-access article distributed under the terms of the Creative Commons Attribution License (CC BY). The use, distribution or reproduction in other forums is permitted, provided the original author(s) and the copyright owner(s) are credited and that the original publication in this journal is cited, in accordance with accepted academic practice. No use, distribution or reproduction is permitted which does not comply with these terms. 